\title{
Endocytosis and Vesicle Recycling at a Ribbon Synapse
}

\author{
Christophe Paillart, ${ }^{1}$ Jian Li, ${ }^{2}$ Gary Matthews, ${ }^{1}$ and Peter Sterling ${ }^{2}$ \\ ${ }^{1}$ Department of Neurobiology and Behavior, State University of New York at Stony Brook, Stony Brook, New York 11794, and ${ }^{2}$ Department of Neuroscience, \\ University of Pennsylvania, Philadelphia, Pennsylvania 19104-6058
}

At ribbon synapses, where exocytosis is regulated by graded depolarization, vesicles can fuse very rapidly with the plasma membrane (complete discharge of the releasable pool in $\sim 200 \mathrm{msec}$ ). Vesicles are also retrieved very rapidly (time constant of $\sim 1 \mathrm{sec}$ ), leading us to wonder whether their retrieval uses an unusual mechanism. To study this, we exposed isolated bipolar neurons from goldfish retina to cationized ferritin. This electron-dense marker uniformly decorated the cell membrane and was carried into the cell during membrane retrieval. Endocytosis was activity-dependent and restricted to the synaptic terminal. The labeling pattern was consistent with direct retrieval from the plasma membrane of large, uncoated endosomes 60-200 $\mathrm{nm}$ in diameter. Even after extensive synaptic activity lasting several minutes, most of the ferritin remained in large endosomes and was present in only $\sim 10 \%$ of the small vesicles that constitute the reserve pool. By contrast, after brief stimulation at a conventional terminal, ferritin did not reside in endosomes but was present in $\sim 63 \%$ of the small vesicles. We suggest that the bipolar ribbon synapse sustains its rapid exocytosis by retrieving membrane in larger "bites" than the clathrin-dependent mechanism thought to dominate at conventional synapses. The resulting large endosomes bud off small vesicles, which reenter the reserve pool and finally the releasable pool.

Key words: bipolar cell; transmitter release; goldfish; ferritin tracer; endocytosis, synaptic vesicles

\section{Introduction}

At a conventional synapse each vesicle that fuses with the plasma membrane is promptly retrieved by endocytosis. The process is thought to begin with clathrin-dependent, inward budding of a membrane bleb similar in size to the original vesicle, followed by pinching off assisted by the GTPase dynamin (for review, see Cremona and de Camilli, 1997; Schmid et al., 1998; Brodin et al., 2000; but also see Palfrey and Artalejo, 1998). The retrieved vesicle is refilled with transmitter and returned within minutes to the active zone for re-release (Betz and Bewick, 1992; Ryan et al., 1993). Such direct recycling is essential to maintain synaptic transmission because the reserve pool of vesicles is modest. Therefore, disrupting endocytosis soon depletes the pool of releasable vesicles, as occurs for example in the Drosophila dynamin mutant shibire (Kosaka and Ikeda, 1983; Delgado et al., 2000).

The ribbon synapse of a retinal bipolar neuron has been shown functionally to exhibit compensatory endocytosis, which restores membrane surface area to baseline with a time constant of 1-2 sec after a bout of exocytosis (von Gersdorff and Matthews, 1994a; Heidelberger, 2001). But this process appears to differ from the conventional mechanism. First, unlike a conventional synapse, in which calcium elevation either has no effect ( $\mathrm{Ra}-$ maswami et al., 1994; Wu and Betz, 1996) or facilitates endocytosis (Klingauf et al., 1998; Marks and McMahon, 1998; Sankaranarayanan and Ryan, 2001), calcium at the bipolar terminal inhibits endocytosis (von Gersdorff and Matthews, 1994b; Rouze

Received Dec. 10, 2002; revised Feb. 24, 2003; accepted Feb. 25, 2003.

This work was supported by National Institutes of Health Grants EY03821 (G.M.) and EY08124 (P.S.). We thank Sally Shrom for contributing to the electron microscopy and Sharron Fina for preparing the figures and this manuscript.

Correspondence should be addressed to Dr. Peter Sterling, 123 Anatomy/Chemistry Building, Department of Neuroscience, University of Pennsylvania, Philadelphia, PA 19104-6058. E-mail: peter@retina.anatomy.upenn.edu.

C. Paillart's present address: Department of Physiology, University of California, San Francisco, 513 Parnassus Avenue, Box 0444, Room S-762, San Francisco, CA 94143-0444.

Copyright $\odot 2003$ Society for Neuroscience $\quad$ 0270-6474/03/234092-08\$15.00/0 and Schwartz, 1998; Neves and Lagnado, 1999; Heidelberger, 2001). Second, unlike a conventional synapse, in which endocytosis is suppressed by GTPase inhibitors (Takei et al., 1995), endocytosis at the bipolar terminal is unaffected (Heidelberger, 2001). Third, unlike a conventional synapse, the bipolar terminal has a large reserve pool ( $\geq 5 \times 10^{5}$ vesicles) (von Gersdorff et al., 1996), and probably as a consequence, blocking compensatory endocytosis does not soon interrupt exocytosis (Heidelberger et al., 2002).

Given these differences, we wondered whether the compensatory membrane retrieval of the bipolar terminal might use an alternative form of endocytosis. For example, would membrane retrieval occur mainly near the active zone, and would it occur in small bites or large ones? Furthermore, would retrieval involve clathrin, and would vesicles directly recycle to the synaptic ribbon, would they be reincorporated into the large reserve pool, or would they be recycled to the soma for degradation?

To approach these questions, we decorated the plasma membrane of isolated bipolar cells with cationized ferritin (TownesAnderson et al., 1988). In the continued presence of ferritin, we then activated the terminal, either by enabling spontaneous calcium action potentials or by elevating external potassium (while monitoring activity with calcium imaging), and fixed the cell with aldehydes. Electron microscopy showed that ferritin-labeled membrane is endocytosed in rather large bites that only later give rise to synaptic vesicles. These vesicles appear to reenter the reserve pool from which the ribbon is reloaded.

\section{Materials and Methods}

$\left[\mathrm{Ca}^{2+}\right]_{i}$ measurements. Single bipolar neurons were isolated by mechanical trituration after the enzymatic digestion of goldfish retina, as described previously (Heidelberger and Matthews, 1992). We studied omega neuron (ON)-type bipolar cells, which are characterized by a large, bulbous synaptic terminal. The external solution consisted of (in $\mathrm{mM}$ ): $115 \mathrm{NaCl}, 2.5 \mathrm{KCl}, 1.6 \mathrm{MgCl}_{2}, 2.5 \mathrm{CaCl}_{2}, 10$ glucose, and 10 HEPES, 
$\mathrm{pH}$ 7.4. For the measurement of $\left[\mathrm{Ca}^{2+}\right]_{\mathrm{i}}$, cells were incubated for $8-10$ min in a solution containing $5 \mu \mathrm{M}$ fura-2 AM (Molecular Probes, Eugene, OR), followed by $25 \mathrm{~min}$ in fura-free solution. $\left[\mathrm{Ca}^{2+}\right]_{\mathrm{i}}$ was calculated from the ratio of the emitted light at two excitation wavelengths (Grynkiewicz et al., 1985), using calibration constants determined by dialyzing cells with highly buffered, known concentrations of $\mathrm{Ca}^{2+}$ (Heidelberger and Matthews 1992).

To prevent the firing of spontaneous calcium action potentials (Zenisek and Matthews, 1998), all solutions up to the beginning of recording contained 1-5 $\mu \mathrm{M}$ muscimol, which hyperpolarized cells by activating $\mathrm{GABA}_{\mathrm{A}}$ chloride conductance. To initiate stimulation, the bath was exchanged with external solution lacking muscimol and containing 0.25 $\mathrm{mg} / \mathrm{ml}$ cationized ferritin (CF) conjugated with fluorescein (Molecular Probes). Activity was monitored by means of the changes in calcium in the synaptic terminal, initiated by spontaneous calcium action potentials. In some experiments, cells were maintained in muscimolcontaining solution and were stimulated by local superfusion with muscimol-free, high-potassium solution $(80 \mathrm{~mm} \mathrm{KCl}$, replacing $\mathrm{NaCl})$ in the continuous presence of $0.25 \mathrm{mg} / \mathrm{ml} \mathrm{CF}$. The high-potassium solution was applied via an application pipette placed under visual control near the terminal, and the application was terminated by removing the application pipette as soon as calcium was observed to increase (application duration typically $\sim 1 \mathrm{sec}$ ).

Electron microscopy. For electron microscopy, a bipolar neuron attached to a film of Aclar was recorded using fura- 2 imaging and then fixed by local superfusion with $2.5 \%$ paraformaldehyde plus $2.5 \%$ glutaraldehyde in $0.1 \mathrm{M}$ phosphate buffer from an application pipette with a tip diameter of $\sim 20 \mu \mathrm{m}$ that was placed within $15 \mu \mathrm{m}$ of the cell. After $10-30 \mathrm{sec}$, the bath fluid was replaced with fixative, a rectangular mark was etched into the Aclar film around the recorded cell using the superfusion pipette, and the dish was placed at $4^{\circ} \mathrm{C}$ overnight. Cells were then additionally fixed in $1 \% \mathrm{OsO}_{4}$ plus $1.5 \% \mathrm{~K}$ ferrocyanide in $0.1 \mathrm{M}$ phosphate buffer, dehydrated, and embedded in Embed 812. The embedded sample was peeled from the Aclar film, glued to an Epon blank, and sectioned serially at $\sim 90 \mathrm{~nm}$. The rectangular mark etched into the Aclar after recording left a corresponding mark in the cured embedding medium to guide sectioning. Localization of the recorded cell was also aided by a reference grid printed onto the Aclar substrate using a laser printer. Sequences of 5-10 sections were mounted on formvar-coated slot grids, stained in methanolic uranyl, and then photographed in an electron microscope $(120 \mathrm{kV})$ at $10,000 \times$. Photos or negatives were then digitized and analyzed using NIH Image or Adobe Photoshop (Adobe Systems, San Jose, CA) software.

Ferritin-labeled structures were identified visually at high magnification, based on the distinctive size and high electron-opacity of ferritin molecules (Fig. 1 A, inset). Other electron-dense objects, such as glycogen granules and uranyl-labeled membrane, lacked the characteristic appearance of ferritin. To preserve the maximum distinction between dark ferritin particles and other dark objects, we scored photos without enhancing contrast. Physiological experiments and embedding for electron microscopy were performed at the State University of New York at Stony Brook, but the electron micrographs were scored at the University of Pennsylvania by an observer (J.L.) who was unaware of the experimental details for each cell until after counts were complete.

\section{Results}

Activity-dependent endocytosis is specific to the synaptic terminal

To study membrane retrieval after calcium-triggered exocytosis, we added the electron-dense tracer cationized ferritin to the medium bathing isolated bipolar neurons to decorate the plasma membranes. Exocytosis was evoked by allowing ON bipolar cells to fire spontaneous calcium action potentials (Zenisek and Matthews, 1998), which were monitored by measuring the accompanying rise in $\left[\mathrm{Ca}^{2+}\right]_{\mathrm{i}}$ within the synaptic terminal. Afterward, we fixed the recorded cell and sectioned it for electron microscopy to locate and identify the ferritin-labeled endocytic structures. The example in Figure $1 A$ shows calcium action potential activity
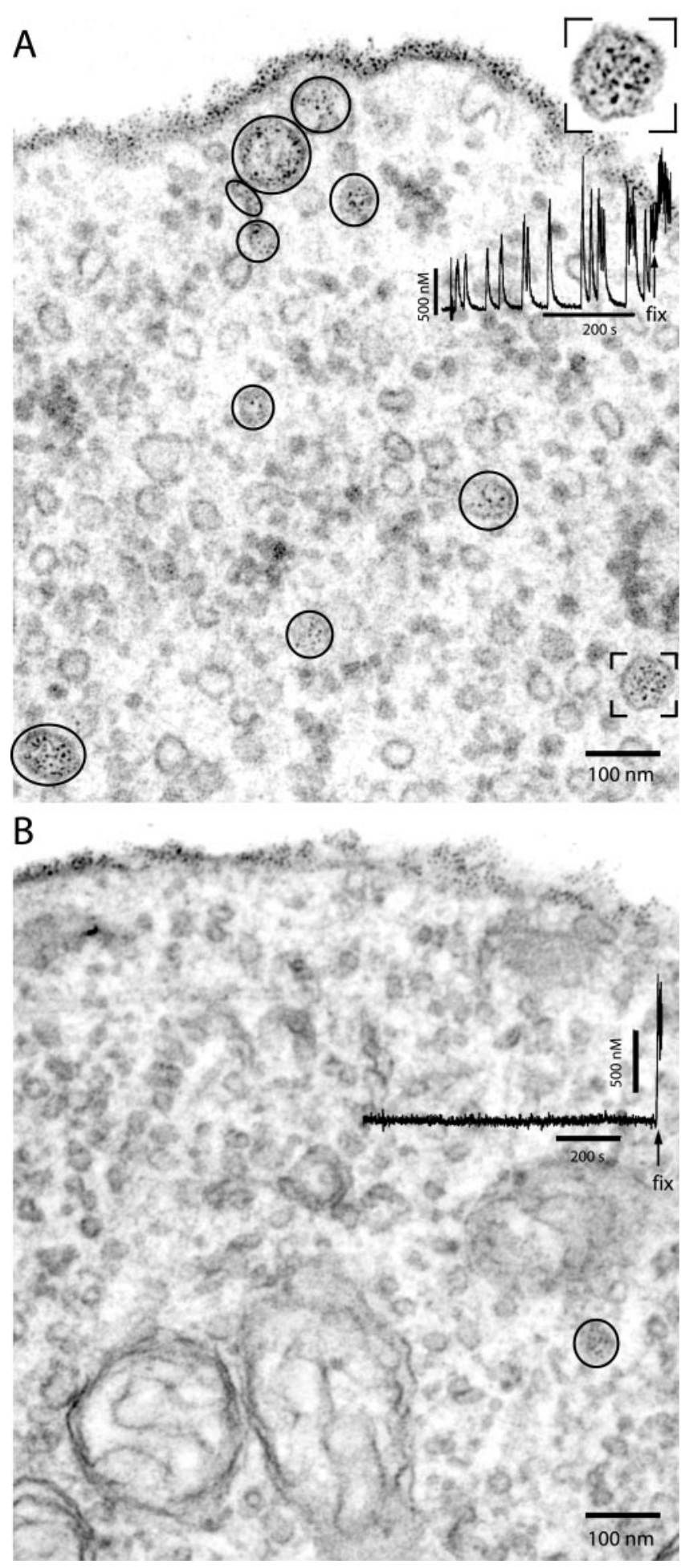

Figure 1. After calcium-triggered exocytosis, plasma membrane was retrieved into large endosomes. $A$, The external surface of isolated bipolar terminal was decorated with cationic ferritin. The terminal fired spontaneous calcium spikes for several minutes (overlay) and was then fixed. Ferritin was internalized in numerous endosomes (circled). The bracketed endosome is shown in the inset with additional magnification $(1.7 \times)$ to better demonstrate the ferritin labeling. $B$, Similar decoration of plasma membrane, but calcium spiking was suppressed by muscimol (overlay). The uptake of ferritin was sparse.

(overlay) in a bipolar cell that was fixed after 8 min of activity. The electron micrograph shows ferritin particles uniformly distributed on the outer face of the plasma membrane and also distributed extensively within the terminal, in endosomes. 
To determine whether the observed ferritin uptake was activity-dependent, we blocked spontaneous firing by including 5 $\mu \mathrm{M}$ muscimol in the bath. Muscimol silences the bipolar cell by hyperpolarizing via a $\mathrm{GABA}_{\mathrm{A}}$ chloride conductance (Zenisek and Matthews, 1998). The example in Figure $1 B$ showed no calcium action potential activity (overlay), and although the electron micrograph shows dense decoration of the plasma membrane, there was little ferritin uptake. In four active cells the average CF density within the terminal was $97 \pm 18$ particles $/ \mu \mathrm{m}^{2}$, whereas in four silent cells the average CF density was $8 \pm 4$ particles $/ \mu \mathrm{m}^{2}$. Thus, ferritin uptake in the synaptic terminal was dramatically increased by calcium action potential activity, which demonstrates that ferritin-labeled endosomes indeed result from activity-dependent endocytosis.

We next asked whether this endocytosis represents synaptic vesicle cycling. If so, it should be restricted to the synaptic terminal. Indeed, the extensive ferritin uptake in cells that fired spontaneous calcium action potentials occurred only in the synaptic terminal (Fig. 2D) and not in the axon or soma (Figs. 2 B,C). Cells silenced with muscimol showed little ferritin uptake in any part of the cell (data not shown), which further confirms that ferritin did not induce significant internalization by itself. Thus, endocytic activity apparently serves to retrieve synaptic vesicle membrane after calcium-triggered fusion with the plasma membrane (von Gersdorff and Matthews, 1994a,b).

\section{Ferritin-labeled large endosomes and some synaptic vesicles}

Most ferritin was found in membrane-bound structures substantially larger than synaptic vesicles (Figs. $1 A, 2,3 A$ ). However, occasionally ferritin was also observed in synaptic vesicles (Fig. $3 A$, arrowheads). Unlabeled synaptic vesicles averaged $30 \pm 6 \mathrm{~nm}$ in diameter (mean $\pm \mathrm{SD} ; n=844$ ), with a narrow range of $20-50$ $\mathrm{nm}$ (Fig. 3B), which agrees with a previous report (von Gersdorff et al., 1996). In contrast, ferritin-labeled endocytic structures averaged $74 \pm 86 \mathrm{~nm}(n=367)$, with a broad range of $20-500 \mathrm{~nm}$ (Fig. 3B).

The relative paucity of label in a structure the size of a synaptic vesicle might simply reflect its small surface area. However, ferritin-labeled vesicles with a diameter of $30 \mathrm{~nm}$ (i.e., the average vesicle diameter) contained an average of 3.2 ferritin particles ( $n=75$ vesicles), which suggests that $<5 \%$ of endocytosed vesicles would fail to contain ferritin for statistical reasons (assuming Poisson statistics and a uniform distribution of ferritin). Also, the observed density of ferritin in endocytic structures $(\sim 1 / 1000$ $\mathrm{nm}^{2}$ ) agrees approximately with the density of ferritin on the plasma membrane (roughly $2-4 / 1000 \mathrm{~nm}^{2}$, assuming a section thickness of $90 \mathrm{~nm}$ ). Finally, as discussed later, ferritin-labeled synaptic vesicles were common at a conventional amacrine-cell synaptic terminal. Taken together, these observations suggest that the rarity of labeled vesicles in the bipolar terminal is not caused by selective retention of ferritin in large structures or its selective exclusion from small vesicles. Thus, we conclude that the sparse labeling of structures the size of synaptic vesicles in bipolar terminals reflects neither geometrical nor statistical constraints and instead represents the nature of the endocytic process.

Do the large ferritin-containing structures represent the transport of ferritin into a preexisting compartment that receives recently endocytosed membrane? Or do they arise de novo in the stimulated terminal? To answer this, we examined five silent terminals and looked for unlabeled structures that resembled the ferritin-containing structures common in stimulated cells. In unstimulated cells 1750 membrane-bound objects located within 1

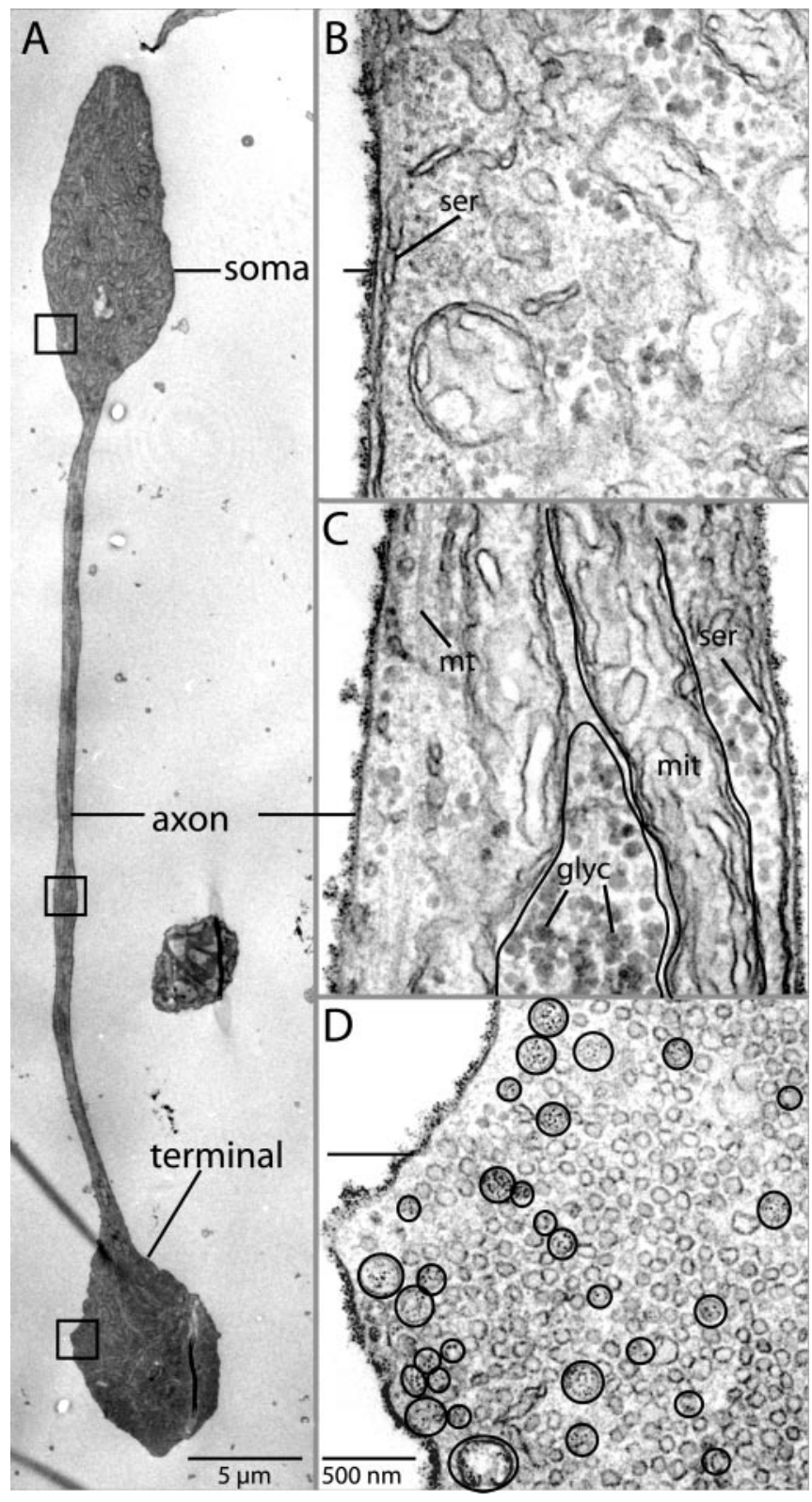

Figure 2. Endocytosis was restricted to the synaptic terminal. $A$, Whole bipolar cell; boxed regions are magnified in $B-D . B, C$, The soma and axon were devoid of ferritin. Note the smooth endoplasmic reticulum (ser) associated with the plasma membrane. mt, Microtubule; mit, mitochondrion; glyc, glycogen. D, The synaptic terminal contained numerous labeled endosomes (circled), mostly larger than unlabeled synaptic vesicles.

$\mu \mathrm{m}$ of the plasma membrane (the location of most labeled endosomes in stimulated cells), only $3.3 \%$ were $>50 \mathrm{~nm}$ in diameter. That is, unstimulated terminals gave no hint of a stable endosome compartment other than synaptic vesicles. Apparently, the larger labeled endosomes (Fig. 3B) represent a transient compartment that arises after stimulated exocytosis or endocytosis.

\section{Labeled synaptic vesicles in the releasable and reserve pools} We next focused on synaptic vesicles that were labeled by ferritin, to estimate the degree to which synaptic vesicles recycle and to determine which vesicle populations are labeled. Synaptic vesicles were defined as having a diameter of $50 \mathrm{~nm}$ or less (Fig. 3B). We compared labeling of vesicles associated with synaptic ribbons (releasable pool) with that of vesicles in the rest of the 


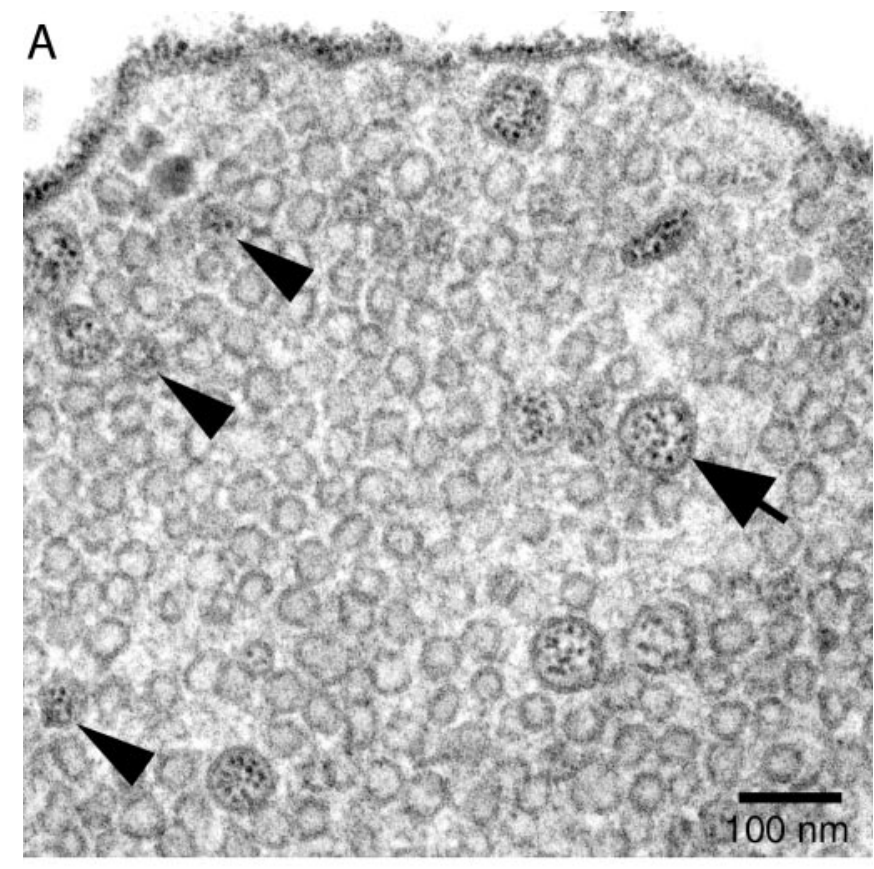

B

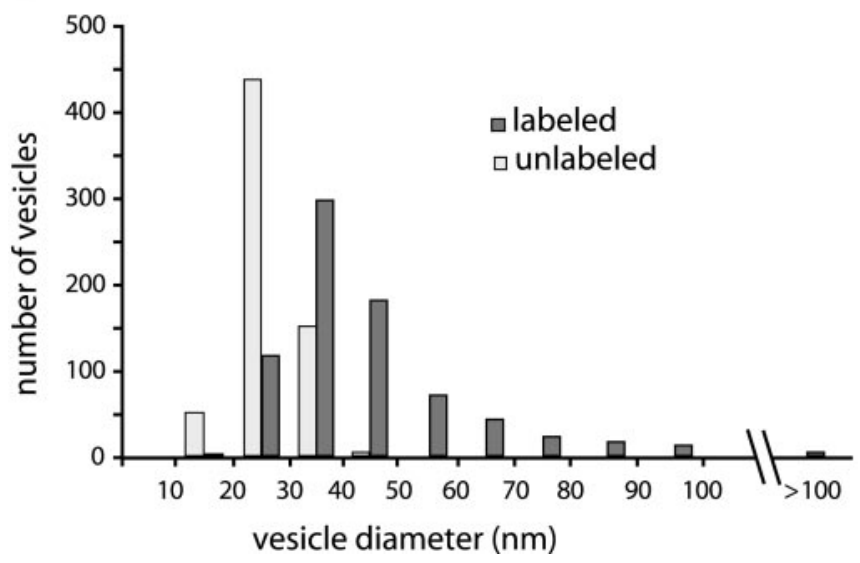

Figure 3. Some labeled endosomes resemble synaptic vesicles, whereas others are much larger. $A$, Arrowheads mark a selection of small labeled vesicles; arrows mark selected larger labeled endosomes. $B$, Histograms compare the single distributions of labeled and unlabeled membrane structures. The cell fired spontaneously for $11 \mathrm{~min}$ before fixation.

terminal (reserve pool). Vesicles were considered to be ribbonassociated if they fell within a circle centered on the ribbon and twice its diameter. Vesicles in the reserve pool were chosen by moving the same circle $1 \mu \mathrm{m}$ away from the ribbon.

After $11 \mathrm{~min}$ of spontaneous calcium action potential activity, some labeled endosomes were associated with the synaptic ribbon and sometimes appeared to bud off a labeled vesicle (Fig. $4 A$ ). Labeled vesicles were themselves sometimes associated with a synaptic ribbon (Fig. $4 B$ ). Overall, 30 of 249 ribbon-associated vesicles $(12 \%)$ contained ferritin $(n=10$ ribbons). In the same synaptic terminal, 26 of 267 synaptic vesicles from the reserve pool (10\%) contained ferritin. Thus, recycled vesicles (i.e., ferritin-labeled) did not appear to move selectively to the ribbon. Furthermore, even after extensive activity lasting several minutes, labeled structures the size of synaptic vesicles were relatively sparse, and most internalized ferritin was sequestered in larger endosomes. This pattern contrasts sharply with a conventional synaptic terminal, which exhibited extensive synaptic vesicle labeling after weak stimulation (see Fig. 8).

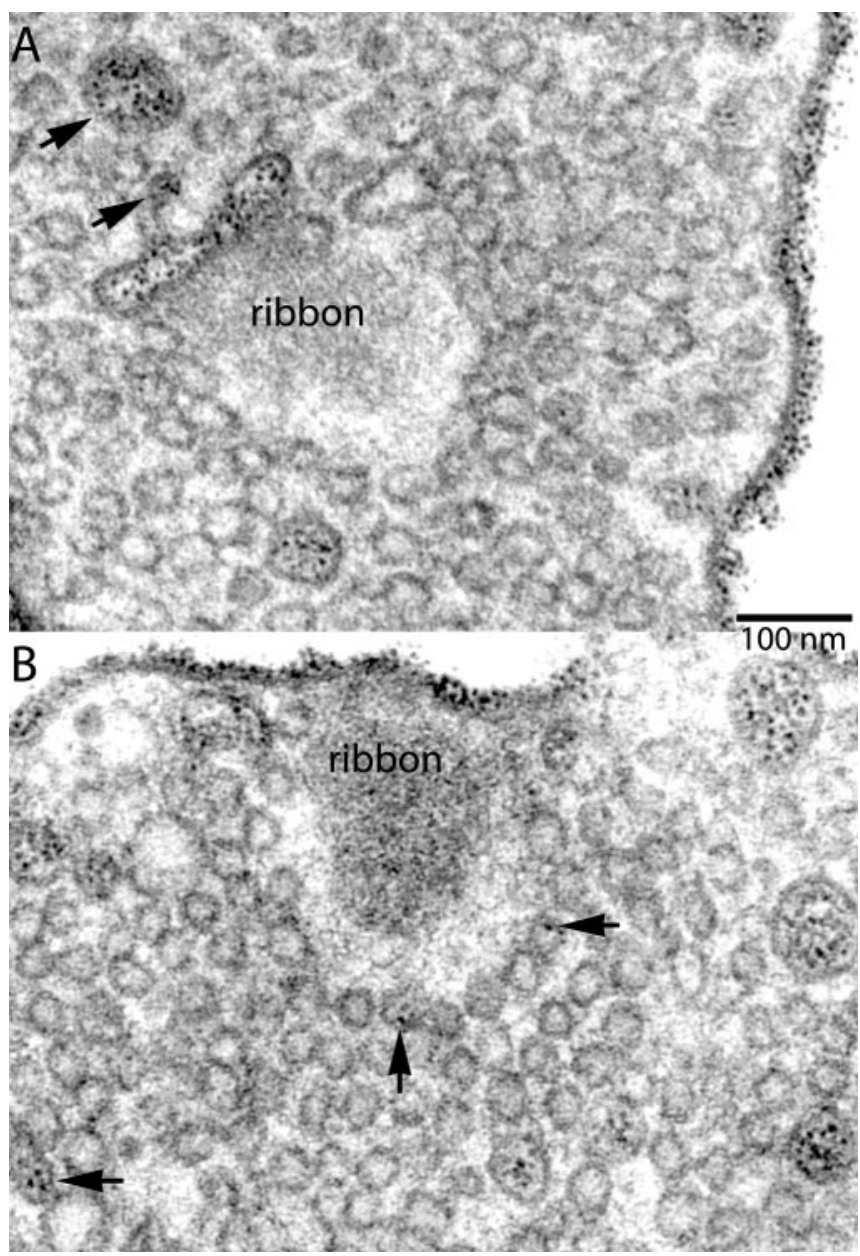

Figure 4. Labeled synaptic vesicles return to the ribbon. A, A labeled endosome associates with a ribbon and appears to bud off a small, labeled vesicle (arrows). This section does not show the attachment of the ribbon to the plasma membrane. $B$, Two vesicles containing ferritin (arrows) tether to a ribbon.

Stimulated terminals often showed spherical, ferritin-labeled endosomes, apparently forming as invaginations of the plasma membrane. Figure 5 shows one invagination at an early stage (right arrow) and another at a later stage, just pinching off from the plasma membrane (left arrow). These endosomes are at least threefold larger in diameter than a synaptic vesicle, but they are confined within one or two sections. Therefore, they must be not much greater in diameter than the section thickness (Fig. 5A-C) $(\sim 80 \mathrm{~nm})$, and certainly less than the diameter of two sections $(160 \mathrm{~nm})$. No membrane coats were visible on any ferritinlabeled endocytic structures, regardless of their size. Such structures suggest that the membrane endosomes larger than synaptic vesicles represent the initial step of endocytosis, rather than a product of small endocytic vesicles that later fuse. This is consistent with membrane retrieval via large, uncoated endosomes, instead of via smaller, coated vesicles. However, the absence of coated vesicles might reflect a high rate of uncoating, so that few endosomes were caught in the coated state.

\section{Uptake after brief stimulation}

The experiments using spontaneous calcium action potentials had the advantage that the stimulus was physiologically relevant, but stimulus timing and intensity were not controlled. Also, in 

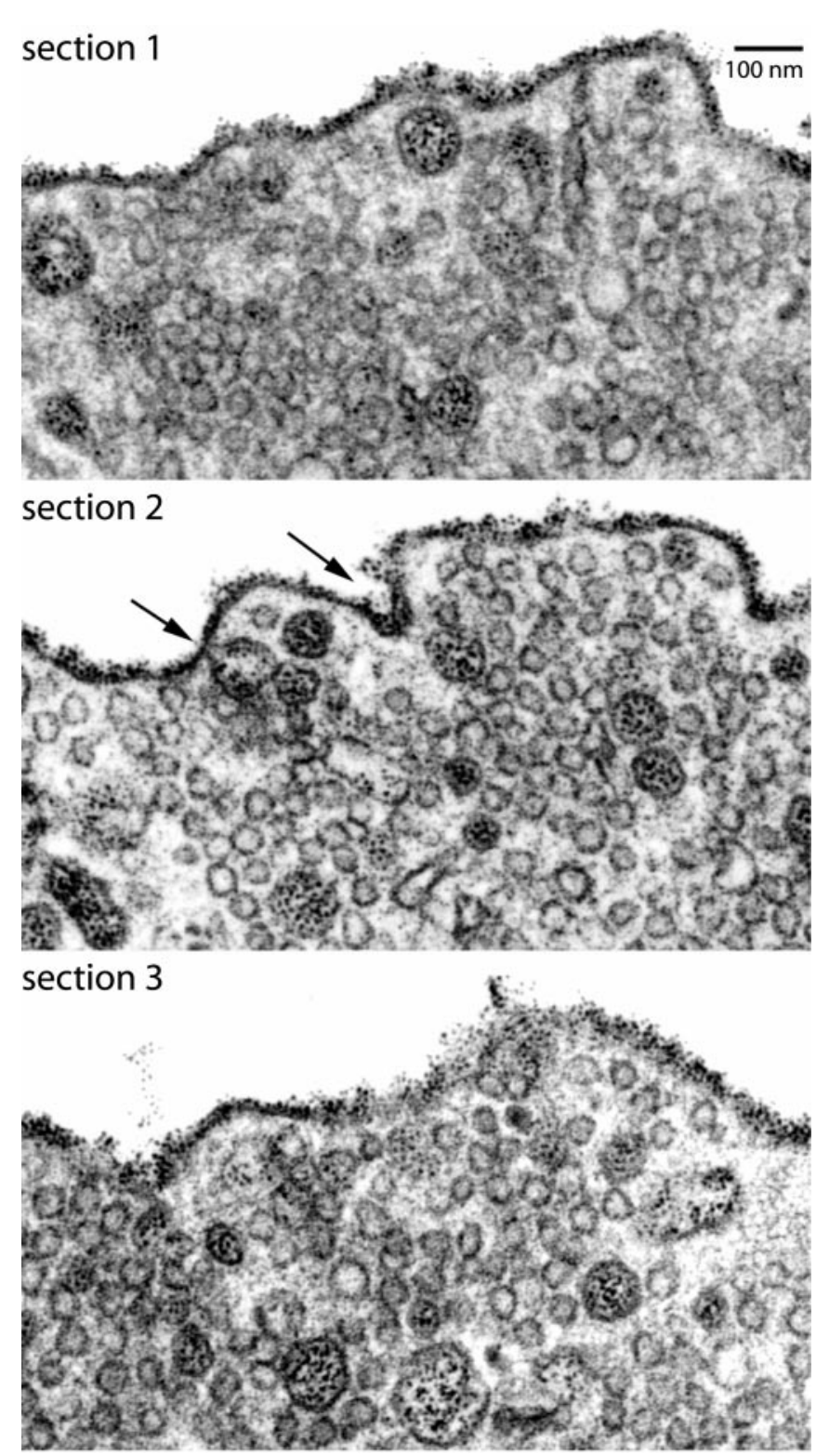

Figure 5. Endosomes forming at the plasma membrane are several times larger than a synaptic vesicle. Three sections from a series are shown, each $\sim 80 \mathrm{~nm}$ thick, with single intervening sections omitted. Section 3 shows an endosome with a neck still attached to the plasma membrane (left arrow), which is absent from sections 1 and 5, implying that the endosome must be $<160 \mathrm{~nm}$ in diameter. The right arrow shows a presumed earlier stage of budding.

these experiments activity continued for several minutes before fixation. Thus, it was unclear whether, during the sustained activation, the large ferritin-labeled endosomes were formed early or late. To examine the earlier stages of activity-dependent uptake, bipolar cells were maintained in external solution containing 1-5 $\mu \mathrm{M}$ muscimol to silence spontaneous activity. Then, cells were briefly superfused with high external potassium to depolarize the cell and trigger a bout of exocytosis. Fixative was then applied by focal superfusion 15-45 sec after high potassium was removed, and the cell was prepared for electron microscopy.

Figure $6 \mathrm{~A}$ shows an example of this type of experiment. As expected for a single cycle of exocytosis-endocytosis, the amount of internalized ferritin was much smaller than in terminals stimulated for minutes with repetitive calcium action potentials. However, just as in those terminals, ferritin in the minimally stimulated terminal was found primarily in structures larger than

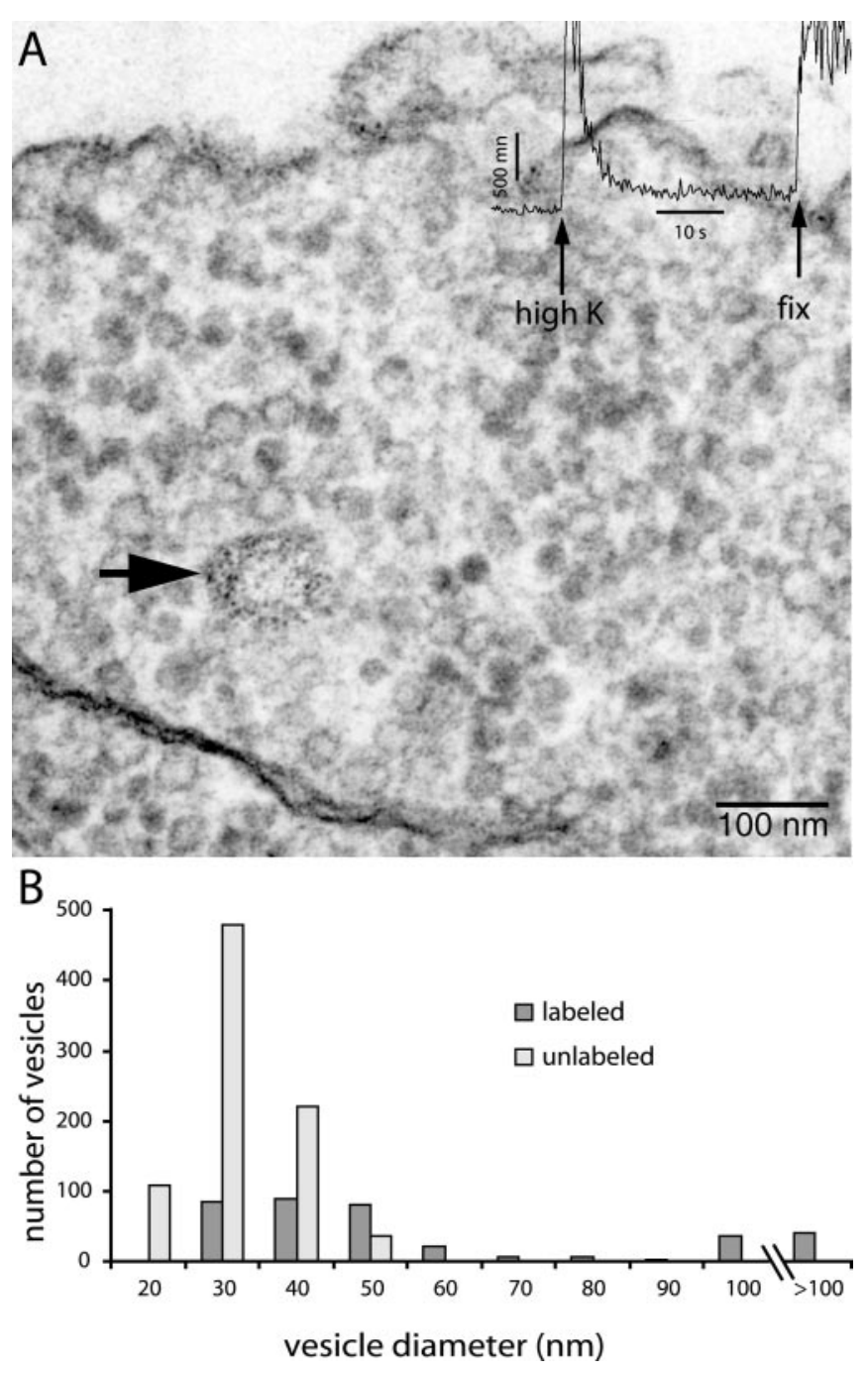

Figure 6. After brief depolarization (elevated potassium), ferritin-labeled endosomes were near the plasma membrane. $A$, This cell was exposed to high potassium (high-K) and fixed after $30 \mathrm{sec}$ ( fix). Labeling was sparser than after prolonged activity (Figs. 1,3) and remained near the plasma membrane. $B$, Labeled structures were mostly larger than synaptic vesicles. This histogram represents summed data from three synaptic terminals fixed after brief depolarization.

synaptic vesicles (Fig. $6 B$ ). The size distribution of labeled structures resembled that observed in experiments with calcium action potentials (Fig. 3B). Thus, large ferritin-labeled structures are not unique to terminals experiencing prolonged, repetitive stimulation, consistent with the notion that the large endosomes represent an early stage of vesicle cycling. Control experiments in which cells were incubated in ferritin but not depolarized with high potassium showed no ferritin uptake, confirming again that the uptake depended on exocytotic activity.

\section{Spatial distribution of ferritin label within the synaptic terminal}

The distribution of label within the synaptic terminal was estimated by counting ferritin particles in successive $1 \mu \mathrm{m}$ shells from the plasma membrane toward the interior of the terminal. Cells depolarized briefly by high potassium showed ferritinlabeled structures mainly near the plasma membrane, whereas terminals stimulated more extensively showed labeled structures 


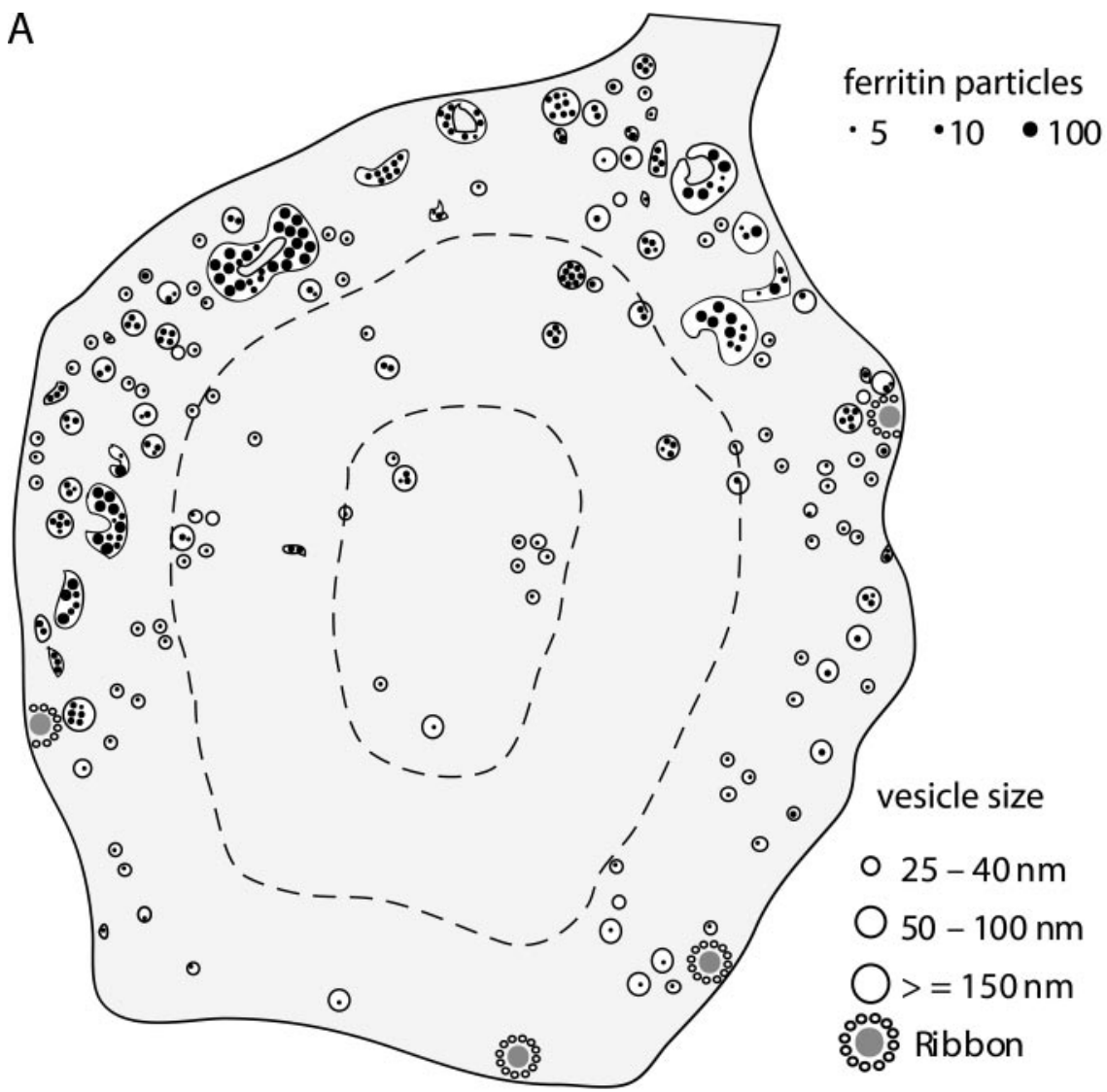

B
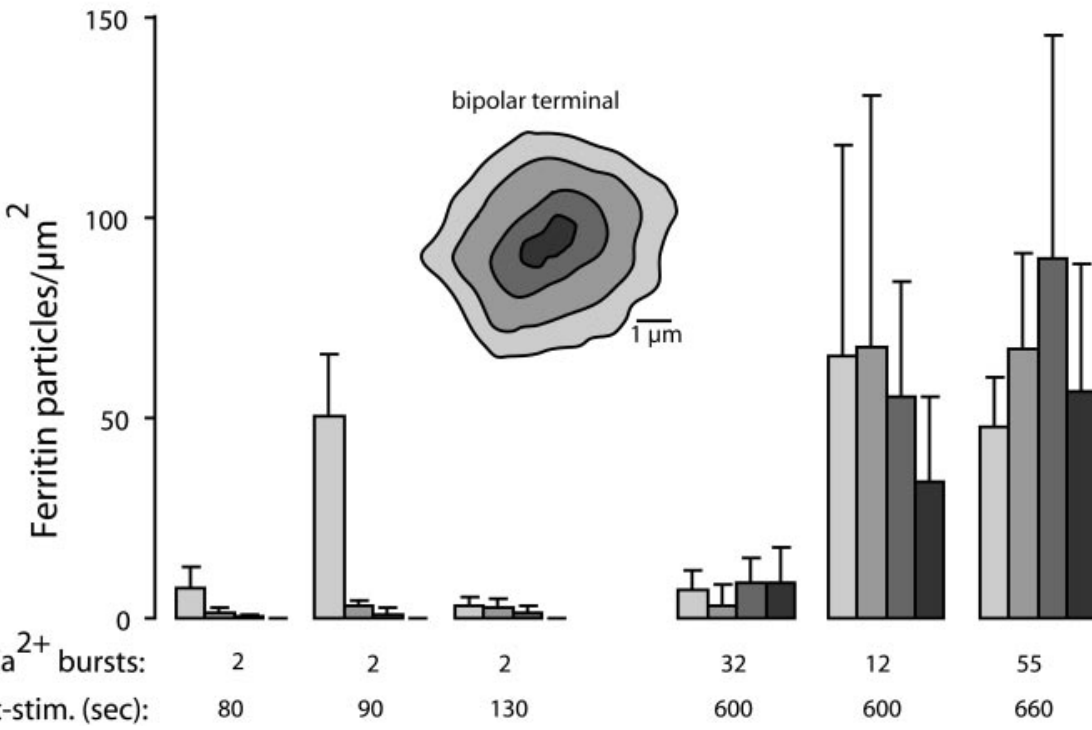

Figure 7. After prolonged spiking, labeled endosomes move from the plasma membrane to distribute throughout the terminal. $A$, Diagram summarizes the distribution of labeled endosomes reconstructed from eight serial sections in a bipolar terminal that fired calcium spikes for 11 min before fixation. Ferritin concentrated in large endosomes (up to 1800 particles), which moved from the plasma membrane toward the cell interior. Some large, heavily labeled endosomes were associated with synaptic ribbons. $B$, In six experiments, ferritin increased with spiking, and endosomes gradually distributed throughout the terminal. The number of calcium bursts indicates the amount of synaptic activity, and the poststimulus incubation period represents the time from the onset of activity to fixation. The three cells on the left were stimulated by brief applications of high external potassium, and the three on the right were stimulated more extensively by spontaneous calcium action potentials during a more prolonged incubation period. Different shades of gray represent positions within the terminal, as indicated in the inset.

throughout the terminal (Fig. $7 A$ ). Figure $7 B$ quantifies this, showing for three terminals stimulated by two brief applications of high potassium, that ferritin was distributed mainly in the outer shells. In contrast, in three terminals stimulated for longer times by multiple calcium action potentials, ferritin-labeled structures were more uniformly distributed across shells (Fig. $7 \mathrm{~B})$. This pattern suggests that ferritinlabeled endosomes depart from their origin at the plasma membrane on a time scale of several minutes, but not within a few tens of seconds.

Figure 7 also demonstrates that the amount of ferritin uptake increased with the amount of stimulation, which was quantified either by the total incubation time in CF before fixation, or by an index of the amount of calcium elevation during the experiment. One notable exception was a cell in which calcium remained elevated throughout the 8 min incubation period, with no periods during which $\left[\mathrm{Ca}^{2+}\right]_{i}$ returned to baseline. In this terminal, despite the extensive elevation of $\left[\mathrm{Ca}^{2+}\right]_{i}$, there was little ferritin uptake. This behavior is consistent with the calcium-dependent inhibition of endocytosis in bipolar terminals (von Gersdorff and Matthews, 1994b; Rouze and Schwartz, 1998; Neves and Lagnado, 1999; Heidelberger, 2001), which prevents membrane retrieval after a stimulus until $\left[\mathrm{Ca}^{2+}\right]_{\mathrm{i}}$ returns to near baseline.

\section{Ferritin uptake in a conventional synaptic terminal}

Amacrine cells form numerous conventional synapses on the giant bipolar terminal (Marc et al., 1978; Yazulla, 1981), but these amacrine synapses were typically lost during our dissociation procedure. However, in one experiment a conventional amacrine terminal remained adherent on the bipolar terminal and was stimulated twice along with the bipolar terminal by brief, high-potassium depolarizations (Fig. 8A), followed by fixation $140 \mathrm{sec}$ after the second stimulus. We include this single, fortuitous observation because labeling in the bipolar and amacrine terminals was strikingly different. The bipolar cell, as expected for minimal stimulation, was sparsely labeled (only 5 of 536 vesicles in an area comparable to the amacrine terminal; i.e., $<1 \%)$. The amacrine terminal was densely labeled (499 of 789 vesicles contained ferritin, 63\%) (Fig. 8B). This suggests that conventional synaptic terminals rely on vesicle recycling to replenish vesicle pools, whereas the giant ribbon-type terminal relies on its huge stock of reserve vesicles. Also, the size distribution of labeled vesicles in the amacrine terminal coincided with the size distribution of synaptic vesicles (Fig. $8 \mathrm{~B}$ ), which contrasts with the large endosomes observed in 


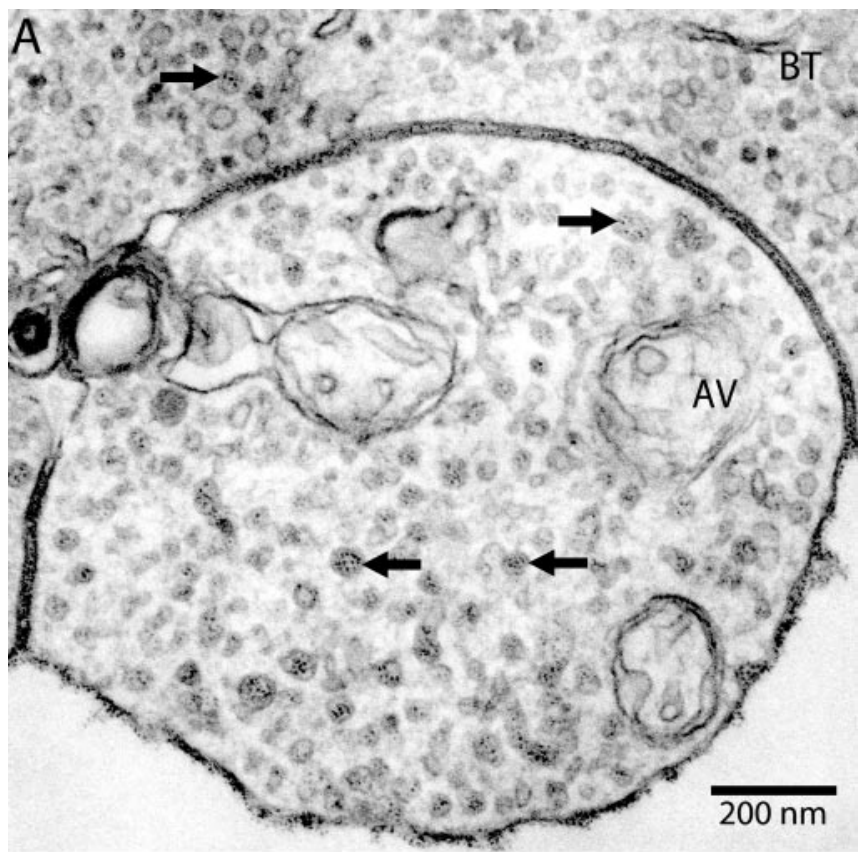

B

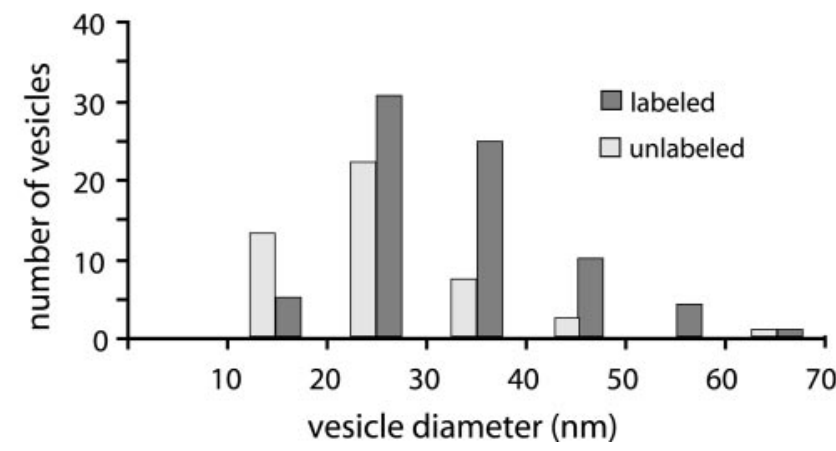

Figure 8. Weak stimulation at a conventional synapse (amacrine cell) labeled most of the reserve pool of synaptic vesicles. A, Amacrine varicosity adhered to a bipolar terminal (BT) and was decorated by ferritin, even in the synaptic cleft. The terminals were stimulated twice by brief, high-potassium depolarization and fixed $180 \mathrm{sec}$ after the first stimulus. In the bipolar terminal, labeling was sparse, whereas in the amacrine varicosity (AV), labeling was present in nearly two-thirds of the vesicles. $B$, In the amacrine varicosity, labeled and unlabeled vesicles show the same size distribution.

bipolar terminals (Figs. 3B, 6B). This difference both highlights the distinctions between conventional amacrine terminals and ribbon-type bipolar terminals and demonstrates that large endosomes are not a necessary feature of the handling of ferritindecorated, endocytosed membrane. No coated vesicles were observed in either the bipolar or the amacrine terminal; however, the long delay $(140 \mathrm{sec})$ between stimulation and fixation in this instance makes it less likely that transiently coated vesicles would be caught.

\section{Discussion}

The endocytosis revealed here by the uptake of cationized ferritin apparently represents cycling of synaptic vesicles in bipolar terminals. First, in unstimulated cells, little ferritin uptake occurred in any cell region. Second, in cells stimulated by calcium action potentials or by high-potassium depolarization, uptake was restricted to the synaptic terminal. Third, ferritin label was observed in synaptic vesicles. Fourth, some of these labeled vesicles were associated with synaptic ribbons, which in bipolar terminals supply releasable vesicles to the active zones. In summary, the endocytic activity revealed by ferritin uptake was specific to the synaptic terminal of stimulated cells and resulted in labeled synaptic vesicles associated with active zones. This pattern is consistent with the activity-dependent fusion, retrieval, and recycling of synaptic vesicles.

Although ferritin label was observed in some synaptic vesicles, most internalized ferritin was found in endosomes several times larger than a synaptic vesicle. This was true both in terminals stimulated repetitively for several minutes and in terminals fixed within seconds after brief depolarization. Also, large endosomes were located preferentially near the plasma membrane (where membrane is retrieved), and structures were observed that are consistent with large endosomes in the process of fission from the plasma membrane. A simple explanation of the overall pattern is that the large endosomes represent the first step in endocytosis, with new synaptic vesicles formed subsequently from the larger structures. In this scheme, the first step in activity-dependent vesicle recycling is retrieval of large bites of membrane (at least 10 times greater membrane area than for retrieved single synaptic vesicles).

This mechanism suggested for the bipolar cell ribbon synapse seems consistent with the finding of Lenzi et al. (2002) that after prolonged depolarization of the saccular hair cell, which also uses ribbon synapses and high release rates, membrane accumulates in large cisterns near the plasma membrane. This mechanism differs from nonribbon synapses, which retrieve small vesicles by clathrin-dependent endocytosis. Although we observed no coated vesicles or pits, we are mindful that compensatory endocytosis in the bipolar terminal is fairly rapid $(\tau \approx 1 \mathrm{sec})$. Thus, if a clathrin-dependent stage were transient, it might escape detection with our relatively slow chemical fixation, and certainly clathrin-coated endosomes (somewhat larger than synaptic vesicles) were observed in the stimulated hair cell (Lenzi et al., 1999, 2002). On the other hand, clathrin-independent endocytosis seems more consistent with the failure of dynamin blockade by GTPase inhibitors to suppress endocytosis in the bipolar terminal (Heidelberger, 2001). In other preparations using the same fixation and electron microscopic procedures, we have observed clathrin coats in cone terminals and certain amacrine terminals, so failure to observe them here is unlikely to represent a technical difficulty.

One unresolved question regarding synaptic vesicles cycling concerns the rate at which vesicle membrane is retired from local cycling and returned to the cell body for degradation. Terminals that fired spontaneous calcium action potentials for as long as 11 min showed no labeled membrane in the axon or soma, suggesting that retrograde membrane traffic must occur on the time scale of hours.

\section{References}

Betz WJ, Bewick GS (1992) Optical analysis of synaptic vesicle recycling at the frog neuromuscular junction. Science 255:200-203.

Brodin L, Low P, Shupliakov O (2000) Sequential steps in clathrinmediated synaptic vesicle endocytosis. Curr Opin Neurobiol 10:312-320.

Cremona O, de Camilli P (1997) Synaptic vesicle endocytosis. Curr Opin Neurobiol 7:323-330.

Delgado R, Maureira C, Oliva C, Kidokoro Y, Labarca P (2000) Size of vesicle pools, rates of mobilization, and recycling at neuromuscular synapses of a Drosophila mutant, shibire. Neuron 28:941-953.

Grynkiewicz G, Poenie M, Tsien RY (1985) A new generation of $\mathrm{Ca}^{2+}$ indicators with greatly improved fluorescence properties. J Biol Chem 260:3440-3450.

Heidelberger R (2001) ATP is required at an early step in compensatory endocytosis in synaptic terminals. J Neurosci 21:6467-6474. 
Heidelberger R, Matthews G (1992) Calcium influx and calcium current in single synaptic terminals of goldfish retinal bipolar neurons. J Physiol (Lond) 447:235-256.

Heidelberger R, Sterling P, Matthews G (2002) Roles of ATP in depletion and replenishment of the releasable pool of synaptic vesicles. J Neurophysiol 88:98-106.

Klingauf J, Kavalali ET, Tsien RW (1998) Kinetics and regulation of fast endocytosis at hippocampal synapses. Nature 394:581-585.

Kosaka T, Ikeda K (1983) Possible temperature-dependent blockage of synaptic vesicle recycling induced by a single gene mutation in Drosophila. J Neurobiol 14:207-225.

Lenzi D, Runyeon JW, Crum J, Ellisman MH, Roberts WM (1999) Synaptic vesicle populations in saccular hair cells reconstructed by electron tomography. J Neurosci 19:119-132.

Lenzi D, Crum J, Ellisman MH, Roberts WM (2002) Depolarization redistributes synaptic membrane and creates a gradient of vesicles on the synaptic body at a ribbon synapse. Neuron 36:649-659.

Marc RE, Stell WK, Bok D, Lam DMK (1978) GABA-ergic pathways in the goldfish retina. J Comp Neurol 182:221-246.

Marks B, McMahon HT (1998) Calcium triggers calcineurin-dependent synaptic vesicle recycling in mammalian nerve terminals. Curr Biol 8:740-749.

Neves G, Lagnado L (1999) The kinetics of exocytosis and endocytosis in the synaptic terminal of goldfish retinal bipolar cells. J Physiol (Lond) 515:181-202.

Palfrey HC, Artalejo CR (1998) Vesicle recycling revisited: rapid endocytosis may be the first step. Neuroscience 83:969-989.

Ramaswami M, Krishnan KS, Kelly RB (1994) Intermediates in synaptic vesicle recycling revealed by optical imaging of Drosophila neuromuscular junctions. Neuron 13:363-375.
Rouze NC, Schwartz EA (1998) Continuous and transient vesicle cycling at a ribbon synapse. J Neurosci 18:8614-8624.

Ryan TA, Reuter H, Wendland B, Schweizer FE, Tsien RW, Smith SJ (1993) The kinetics of synaptic vesicle recycling measured at single presynaptic boutons. Neuron 11:713-724.

Sankaranarayanan S, Ryan TA (2001) Calcium accelerates endocytosis of vSNAREs at hippocampal synapses. Nat Neurosci 4:129-136.

Schmid SL, McNiven MA, de Camilli P (1998) Dynamin and its partners: a progress report. Curr Opin Cell Biol 10:504-512.

Takei K, McPherson PS, Schmid SL, de Camilli P (1995) Tubular membrane invaginations coated by dynamin rings are induced by GTP- $\gamma \mathrm{S}$ in nerve terminals. Nature 374:186-190.

Townes-Anderson E, Dacheux RF, Raviola E (1988) Rod photoreceptors dissociated from the adult rabbit retina. J Neurosci 8:320-331.

von Gersdorff H, Matthews G (1994a) Dynamics of synaptic vesicle fusion and membrane retrieval in synaptic terminals. Nature 367:735-739.

von Gersdorff H, Matthews G (1994b) Inhibition of endocytosis by elevated internal calcium in a synaptic terminal. Nature 370:652-655.

von Gersdorff H, Vardi E, Matthews G, Sterling P (1996) Evidence that vesicles on the synaptic ribbon of retinal bipolar neurons can be rapidly released. Neuron 16:1221-1227.

Wu L-G, Betz WJ (1996) Nerve activity but not intracellular calcium determines the time course of endocytosis at the frog neuromuscular junction. Neuron 17:769-779.

Yazulla S (1981) GABAergic synapses in the goldfish retina: an autoradiographic study of ${ }^{3} \mathrm{H}$-muscimol and ${ }^{3} \mathrm{H}-\mathrm{GABA}$ binding. J Comp Neurol 200:83-93.

Zenisek D, Matthews G (1998) Calcium action potentials in retinal bipolar neurons. Vis Neurosci 15:69-75. 